\title{
A Method to Construct Sets of Commuting Matrices
}

\author{
Hongmei Wang ${ }^{1}$, Guoju Ye ${ }^{1}$, Hao Zhou ${ }^{1} \&$ Bing Liang ${ }^{1}$ \\ ${ }^{1}$ College of Science, Hohai University, Nanjing 210098, P. R. China. \\ Correspondence: Guoju Ye, College of Science, Hohai University, Nanjing 210098, P. R. China. \\ E-mail: yegj@hhu.edu.cn
}

Received: April 21, 2015 Accepted: May 1, 2015 Online Published: May 23, 2015

doi:10.5539/jmr.v7n2p195 URL: http://dx.doi.org/10.5539/jmr.v7n2p195

\begin{abstract}
Using the method of upper and lower solutions, we study the existence of solutions of the hyperbolic equation involving the distributional Henstock-Kurzweil integral. Results presented in this paper are extension of the previous results in the literatures.
\end{abstract}

Keywords: hyperbolic equation; upper and lower solutions; distributional Henstock-Kurzweil integral; distributional derivative.

\section{Introduction}

In the present paper, we consider the existence for solutions of the hyperbolic equation

$$
\left\{\begin{array}{lc}
\frac{\partial^{2} z}{\partial x \partial y}=f\left(x, y, z, \frac{\partial z}{\partial x}, \frac{\partial z}{\partial y}\right), & \text { on } Q \\
z(x, c)=\sigma(x), & a \leq x \leq b, \\
z(a, y)=\tau(y), & c \leq y \leq d,
\end{array}\right.
$$

where $Q=\{(x, y): a<x<b, c<y<d\}, a, c \geq 0, \bar{Q}$ is the closure of $Q, z \in C(\bar{Q}), C(\bar{Q})$ denotes the space of all continuous functions on $\bar{Q}$. $\frac{\partial^{2} z}{\partial x \partial y}$ denotes the mixed distributional derivative of $z$ and $f$ is distributionally Henstock-Kurzweil integrable on $Q$.

The hyperbolic equations have been studied by many contributors. In (A. Alexiewicz \& W. Orlicz (1956)), the authors use the ordinary derivative to discuss the hyperbolic equation. Moreover, authors use the approximate derivative instead of the usual derivative to discuss a special case of hyperbolic equation in (D. Bugajewska \& S. Szufla (1995)). The classical Carathéodory's existence theorem about the special case is discussed in (F. Deblasi \& J. Myjak, (1986)). It is well known that the notion of a distributional derivative is a general concept, including ordinary derivatives and approximate derivatives. In this paper, we use the distributional derivative instead of the approximate derivative and usual derivative to study the hyperbolic equation (1). The distributional HenstockKurzweil integral is defined by the distributional derivative. Hence our results get the corresponding results in (A. Alexiewicz \& W. Orlicz (1956)) by another way.

This paper is organized as follows. In section 2, we define the distributional Henstock-Kurzweil integral in the plane and present its preliminary concepts and properties. In section 3, we apply the method of upper and lower solutions to verify the existence of solutions of hyperbolic equation (1) under weaker hypotheses. In Section 4, two examples are also worked out to illustrate our results.

\section{The Distributional Henstock-Kurzweil Integral}

In this section, we present the definition and some basic properties of the distributional Henstock-Kurzweil integral.

Denote $\mathcal{D}(\Omega)=C_{c}^{\infty}=\left\{\phi: \Omega \rightarrow R \mid \phi \in C^{\infty}(\Omega)\right.$ and $\phi$ has compact support in $\left.\Omega\right\}$ where $\Omega$ denotes the open rectangle $(a, b) \times(c, d)$ in the plane $R^{2}, a, b, c, d \in R, a<b$ and $c<d$ and the closure of the set on which $\phi$ does not vanish. The continuous linear functional on $D(\Omega)$ are called distribution, the dual space to $\mathcal{D}(\Omega)$ is denoted by $\mathcal{D}^{\prime}(\Omega)$ and its elements are called distributions. Define the generalized derivative $f^{\prime}$ of distribution $f$ as $\left\langle f^{\prime}, \phi\right\rangle=-\left\langle f, \phi^{\prime}\right\rangle$, where $f \in \mathcal{D}^{\prime}(\Omega), f^{\prime} \in \mathcal{D}^{\prime}(\Omega), \phi \in D(\Omega)$. Under this generalized derivative, distribution is any differentiable and any derivative of the distribution is distribution. Through this paper, $\partial=\partial_{x y}=\partial_{y x}$ stand for the mixed distributional derivative and " $\int$ " denotes the $D_{H K}$-integral. 
Note that $B_{C}(\bar{\Omega})=\{F \in C(\bar{\Omega}): F(a, y)=F(x, c)=0, x \in[a, b], y \in[c, d]\}$ is a Banach space with the uniform norm $\|F\|_{\infty}=\max \{|F(x, y)|:(x, y) \in \bar{\Omega}, F(x, y) \in C(\bar{\Omega})\}$.

Now we are able to introduce the definition of $D_{H K}$-integral on $\Omega$.

Definition 1 A distribution $f$ is distributionaly Henstock-Kurzweil integrable on $\bar{\Omega}$ if $f$ is the distributional derivative of a continuous function $F \in B_{C}(\bar{\Omega})$.

The $D_{H K}$-integral of $f$ on $\bar{\Omega}$ is given by

$$
\int_{\bar{\Omega}} f=F(b, d)
$$

Denoted the space of $D_{H K}$-integrable distributions by

$$
D_{H K}(\Omega)=\left\{f \in \mathcal{D}^{\prime}(\Omega) \mid f=\partial F, F \in B_{C}(\bar{\Omega})\right\} .
$$

In the following, we consider the structure of $D_{H K}(\Omega)$. For $f \in D_{H K}(\Omega)$, the norm is defined as the follows:

$$
\|f\|=\sup \left\{\left|\int_{[a, x] \times[c, y]} f\right|:(x, y) \in \bar{\Omega}\right\} .
$$

Lemma 1([D. D. Ang, K. Schmitt \& L. K. Vy,(1997),Theorem 1]) $\left(D_{H K}(\Omega),\|\cdot\|\right)$ is a separable Banach space, isomorphic to $\left(B_{C}(\bar{\Omega}),\|\cdot\|_{\infty}\right)$.

Now we introduce an ordering in the space $D_{H K}(\Omega)$. For $f, g \in D_{H K}(\Omega)$, we say that $f \geq g$ if and only if $f-g$ is a positive measure on $\bar{\Omega}$. We obtain the ordering preserving property of the $D_{H K}$-integral in (Ang et al., 1997), i.e,

$$
\int_{\bar{\Omega}} f \geq \int_{\bar{\Omega}} g
$$

whenever $f \geq g, f, g \in D_{H K}(\Omega)$.

Definition 2 Let $f \in D_{H K}(\Omega), x \in[a, b], y \in[c, d]$. We define

$$
\begin{aligned}
& \partial_{1} F(\cdot, y)=\int_{c}^{y} f(\cdot, v) d v, \\
& \partial_{2} F(x, \cdot)=\int_{a}^{x} f(u, \cdot) d u .
\end{aligned}
$$

From this definition, we know that $\partial_{1} F(\cdot, y) \in D_{H K}((a, b)), \partial_{2} F(x, \cdot) \in D_{H K}((c, d))$.

Now we give the Fubini type theorem for the $D_{H K}$-integral.

Lemma 2 ([D. D. Ang, K. Schmitt \& L. K. Vy,(1997), Theorem 4, Fubini theorem]) For all $f \in D_{H K}(\Omega)$, we have

$$
\int_{\Omega} f=\int_{a}^{b}\left(\int_{c}^{d} f(\cdot, \eta) d \eta\right)=\int_{c}^{d}\left(\int_{a}^{b} f(\xi, \cdot) d \xi\right) .
$$

A sequence $\left\{f_{n}\right\} \subset D_{H K}(\Omega)$ converges strongly to $f \in D_{H K}(\Omega)$ if $\left\|f_{n}-f\right\| \rightarrow 0$ as $n \rightarrow \infty$. This implies that $\lim _{n \rightarrow \infty} \int_{\bar{\Omega}} f_{n}=\int_{\bar{\Omega}} f$. Then we have the following convergence theorem.

Lemma 3 [D. D. Ang, K. Schmitt \& L. K. Vy,(1997),Corollary 5, Dominated convergence theorem of $D_{H K^{-}}$ integral] Let $\left\{f_{n}\right\} \subset D_{H K}(\Omega)$ such that $f_{n} \rightarrow f$ in $\mathcal{D}^{\prime}(\Omega)$. Suppose there exist $f_{-}, f_{+} \in D_{H K}(\Omega)$ satisfying $f_{-} \leq f_{n} \leq$ $f_{+}, \forall n \in N$. Then $f \in D_{H K}(\Omega)$ and $\lim _{n \rightarrow \infty}\left(D_{H K}\right) \int_{\bar{\Omega}} f_{n}=\left(D_{H K}\right) \int_{\bar{\Omega}} f$.

\section{Main Results}

In this section, we shall prove the existence of solutions of the hyperbolic equation (1).

Assume that $f$ satisfies

( $\left.C_{1}\right) f\left(x, y, z, \frac{\partial z}{\partial x}, \frac{\partial z}{\partial y}\right)$ is $D_{H K}$-integrable with respect to $(x, y) \in \bar{Q}$. 
$\left(C_{2}\right) f\left(x, y, z, \frac{\partial z}{\partial x}, \frac{\partial z}{\partial y}\right)$ is increasing with respect to $z \in C(\bar{Q})$ and $\frac{\partial z}{\partial x}, \frac{\partial z}{\partial y} \in D_{H K}(Q)$.

$\left(C_{3}\right)$ There exist $f_{1}, f_{2} \in D_{H K}(Q)$ such that for all $z \in C(\bar{Q})$ and $\frac{\partial z}{\partial x}, \frac{\partial z}{\partial y} \in D_{H K}(Q), f_{1}(\cdot, \cdot) \leq f\left(\cdot, \cdot, z, \frac{\partial z}{\partial x}, \frac{\partial z}{\partial y}\right) \leq$ $f_{2}(\cdot, \cdot)$ on $\bar{Q}$.

We recall that $\left[u_{0}, v_{0}\right]=\left\{z \in C(\bar{Q}): u_{0}(x, y) \leq z(x, y) \leq v_{0}(x, y),(x, y) \in \bar{Q}\right\}$.

Let $E$ be an ordered Banach space, $K$ a nonempty subset of $E$. The mapping $\mathcal{A}: K \rightarrow E$ is increasing if and only if $\mathcal{A} u \leq \mathcal{A} v$, whenever $u, v \in K$ and $u \leq v$. The symbol " $\leq$ " represents the relationship of size in the space of $E$.

Lemma 4([D. Guo, Y. J. Cho \& J. Zhu, (2004), Theorem 3.1.3])Let $u_{0}, v_{0} \in E$ with $u_{0}<v_{0}$ and $T:\left[u_{0}, v_{0}\right] \rightarrow E$ be an increasing mapping satisfying $u_{0} \leq T u_{0}, T v_{0} \leq v_{0}$. If $T\left(\left[u_{0}, v_{0}\right]\right)$ is relatively compact, then $T$ has a maximal fixed point $z^{*}$ and a minimal fixed point $z_{*}$ in $\left[u_{0}, v_{0}\right]$. Moreover,

$$
z_{*}=\lim _{n \rightarrow \infty} u_{n}, z^{*}=\lim _{n \rightarrow \infty} v_{n}
$$

where $u_{n}=T u_{n-1}$ and $v_{n}=T v_{n-1}(n=1,2,3, \cdots)$,

$$
u_{0} \leq u_{1} \leq \cdots \leq u_{n} \leq \cdots \leq z_{*} \leq z^{*} \leq \cdots \leq v_{n} \leq \cdots \leq v_{1} \leq v_{0} .
$$

Now we give the main result of this paper.

Theorem 1Under the assumptions $\left(C_{1}\right)-\left(C_{3}\right)$, there exist monotone sequences $\left\{u_{n}\right\}_{n=0}^{\infty}$ and $\left\{v_{n}\right\}_{n=0}^{\infty}$ on $C(\bar{Q})$ with $u_{0}(x, y)=F_{1}(x, y)+\sigma(x)+\tau(y)-\sigma(a)$ and $v_{0}(x, y)=F_{2}(x, y)+\sigma(x)+\tau(y)-\sigma(a)$ where

$$
F_{1}(x, y)=\int_{a}^{x} \int_{c}^{y} f_{1}(u, v) d v d u, F_{2}(x, y)=\int_{a}^{x} \int_{c}^{y} f_{2}(u, v) d v d u
$$

such that

$$
\lim _{n \rightarrow \infty} u_{n}(x, y)=z_{*}(x, y), \lim _{n \rightarrow \infty} v_{n}(x, y)=z^{*}(x, y),
$$

where $z_{*}$ and $z^{*}$ are the minimal and maximal solutions of the hyperbolic equation (1), respectively.

proof After integral operation of the hyperbolic equation (1), we can get that

$$
z(x, y)+f(x)+f(y)=\int_{a}^{x} \int_{c}^{y} f\left(u, v, z(u, v), \frac{\partial z(u, v)}{\partial u}, \frac{\partial z(u, v)}{\partial v}\right) d v d u .
$$

The insertion $y=c$ and $x=a$ in (5) yields $z(x, c)+f(x)+f(c)=0$ and $z(a, y)+f(a)+f(y)=0$, which together with (1) imply that $f(x)=-f(c)-\sigma(x)$ and $f(y)=-f(a)-\tau(y)$.

So, $z(x, y)=\sigma(x)+\tau(y)+f(c)+f(a)+\int_{a}^{x} \int_{c}^{y} f\left(u, v, z(u, v), \frac{\partial z(u, v)}{\partial u}, \frac{\partial z(u, v)}{\partial v}\right) d v d u$.

Since $f(a)+f(c)=-\sigma(a)=-\tau(c)$,

$$
z(x, y)=\sigma(x)+\tau(y)-\sigma(a)+\int_{a}^{x} \int_{c}^{y} f\left(u, v, z(u, v), \frac{\partial z(u, v)}{\partial u}, \frac{\partial z(u, v)}{\partial v}\right) d v d u .
$$

Compute the mixed partial derivative of $z$ with respect to $x$ and $y$ in (6). Then we can get the hyperbolic equation (1). Therefore, the hyperbolic equation (1) is equivalent to the integral equation (6).

For $(x, y) \in \bar{Q}, z \in C(\bar{Q})$, define a mapping

$$
\mathcal{A} z(x, y):=\int_{a}^{x} \int_{c}^{y} f\left(u, v, z(u, v), \frac{\partial z(u, v)}{\partial u}, \frac{\partial z(u, v)}{\partial v}\right) d v d u+\sigma(x)+\tau(y)-\sigma(a) .
$$

In view of $\left(C_{2}\right), \mathcal{A}$ is increasing.

Now we verify that $\mathcal{A}$ satisfies the hypotheses of Lemma 4 in two steps.

Step 1: $\mathcal{A}:\left[u_{0}, v_{0}\right] \rightarrow\left[u_{0}, v_{0}\right]$. 
Let

$$
F_{1}(x, y)=\int_{a}^{x} \int_{c}^{y} f_{1}(u, v) d v d u, \quad F_{2}(x, y)=\int_{a}^{x} \int_{c}^{y} f_{2}(u, v) d v d u .
$$

Since $f_{1}, f_{2} \in D_{H K}(\bar{Q})$, then the primitives $F_{1}(x, y)$ and $F_{2}(x, y)$ are continuous on $\bar{Q}$.

According to $\left(C_{3}\right)$ and (2), we have

$$
F_{1}(x, y) \leq \int_{a}^{x} \int_{c}^{y} f\left(u, v, z(u, v), \frac{\partial z(u, v)}{\partial u}, \frac{\partial z(u, v)}{\partial v}\right) d v d u \leq F_{2}(x, y) .
$$

It follows from (7) and (8) that

$$
F_{1}(x, y)+\sigma(x)+\tau(y)-\sigma(a) \leq \mathcal{A} z(x, y) \leq F_{2}(x, y)+\sigma(x)+\tau(y)-\sigma(a) .
$$

Denote

$$
\begin{aligned}
u_{0}(x, y) & =F_{1}(x, y)+\sigma(x)+\tau(y)-\sigma(a), \\
v_{0}(x, y) & =F_{2}(x, y)+\sigma(x)+\tau(y)-\sigma(a) .
\end{aligned}
$$

It is clear that $u_{0}(x, y), v_{0}(x, y)$ are continuous on $\bar{Q}$ and

$$
u_{0}(x, y) \leq \mathcal{A} z(x, y) \leq v_{0}(x, y), \forall z \in\left[u_{0}, v_{0}\right] .
$$

Hence, $\mathcal{A}:\left[u_{0}, v_{0}\right] \rightarrow\left[u_{0}, v_{0}\right]$.

Step 2: $\mathcal{A}\left(\left[u_{0}, v_{0}\right]\right)$ is relatively compact.

Let $\left(x_{1}, y_{1}\right),\left(x_{2}, y_{2}\right) \in \bar{Q}$, we have, for each $z \in\left[u_{0}, v_{0}\right]$,

$$
\begin{aligned}
\mathcal{A}(z)\left(x_{1}, y_{1}\right)-\mathcal{A}(z)\left(x_{2}, y_{2}\right)= & \int_{a}^{x_{1}} \int_{c}^{y_{1}} f\left(u, v, z(u, v), \frac{\partial z(u, v)}{\partial u}, \frac{\partial z(u, v)}{\partial v}\right) d v d u \\
& -\int_{a}^{x_{2}} \int_{c}^{y_{2}} f\left(u, v, z(u, v), \frac{\partial z(u, v)}{\partial u}, \frac{\partial z(u, v)}{\partial v}\right) d v d u \\
& +\sigma\left(x_{1}\right)-\sigma\left(x_{2}\right)+\tau\left(y_{1}\right)-\tau\left(y_{2}\right) .
\end{aligned}
$$

For simplicity, we denote

$$
\begin{aligned}
T= & \int_{a}^{x_{1}} \int_{c}^{y_{1}} f\left(u, v, z(u, v), \frac{\partial z(u, v)}{\partial u}, \frac{\partial z(u, v)}{\partial v}\right) d v d u \\
& -\int_{a}^{x_{2}} \int_{c}^{y_{2}} f\left(u, v, z(u, v), \frac{\partial z(u, v)}{\partial u}, \frac{\partial z(u, v)}{\partial v}\right) d v d u .
\end{aligned}
$$

In view of Lemma 2, then

$$
\begin{aligned}
T= & \int_{a}^{x_{2}}\left(\int_{c}^{y_{1}} f\left(u, v, z(u, v), \frac{\partial z(u, v)}{\partial u}, \frac{\partial z(u, v)}{\partial v}\right) d v\right) d u \\
& +\int_{x_{2}}^{x_{1}}\left(\int_{c}^{y_{1}} f\left(u, v, z(u, v), \frac{\partial z(u, v)}{\partial u}, \frac{\partial z(u, v)}{\partial v}\right) d v\right) d u \\
& -\int_{c}^{y_{1}}\left(\int_{a}^{x_{2}} f\left(u, v, z(u, v), \frac{\partial z(u, v)}{\partial u}, \frac{\partial z(u, v)}{\partial v}\right) d u\right) d v \\
& +\int_{y_{2}}^{y_{1}}\left(\int_{a}^{x_{2}} f\left(u, v, z(u, v), \frac{\partial z(u, v)}{\partial u}, \frac{\partial z(u, v)}{\partial v}\right) d u\right) d v \\
= & \int_{x_{2}}^{x_{1}}\left(\int_{c}^{y_{1}} f\left(u, v, z(u, v), \frac{\partial z(u, v)}{\partial u}, \frac{\partial z(u, v)}{\partial v}\right) d v\right) d u \\
& +\int_{y_{2}}^{y_{1}}\left(\int_{a}^{x_{2}} f\left(u, v, z(u, v), \frac{\partial z(u, v)}{\partial u}, \frac{\partial z(u, v)}{\partial v}\right) d u\right) d v .
\end{aligned}
$$


Hence

$$
\begin{aligned}
\mathcal{A}(z)\left(x_{1}, y_{1}\right)-\mathcal{A}(z)\left(x_{2}, y_{2}\right)= & \int_{x_{2}}^{x_{1}}\left(\int_{c}^{y_{1}} f\left(u, v, z(u, v), \frac{\partial z(u, v)}{\partial u}, \frac{\partial z(u, v)}{\partial v}\right) d v\right) d u \\
& +\int_{y_{2}}^{y_{1}}\left(\int_{a}^{x_{2}} f\left(u, v, z(u, v), \frac{\partial z(u, v)}{\partial u}, \frac{\partial z(u, v)}{\partial v}\right) d u\right) d v \\
& +\sigma\left(x_{1}\right)-\sigma\left(x_{2}\right)+\tau\left(y_{1}\right)-\tau\left(y_{2}\right) .
\end{aligned}
$$

By $\left(C_{3}\right)$

$$
\begin{aligned}
\left|\mathcal{A}(z)\left(x_{1}, y_{1}\right)-\mathcal{A}(z)\left(x_{2}, y_{2}\right)\right| \leq & \left|\int_{x_{2}}^{x_{1}}\left(\int_{c}^{y_{1}} f_{1}(u, v) d v\right) d u\right|+\left|\int_{y_{2}}^{y_{1}}\left(\int_{a}^{x_{2}} f_{1}(u, v) d u\right) d v\right| \\
& +\left|\int_{x_{2}}^{x_{1}}\left(\int_{c}^{y_{1}} f_{2}(u, v) d v\right) d u\right|+\left|\int_{y_{2}}^{y_{1}}\left(\int_{a}^{x_{2}} f_{2}(u, v) d u\right) d v\right| \\
& +\left|\sigma\left(x_{1}\right)-\sigma\left(x_{2}\right)\right|+\left|\tau\left(y_{1}\right)-\tau\left(y_{2}\right)\right| .
\end{aligned}
$$

Since $f_{1}, f_{2} \in D_{H K}(Q)$ and $z \in C(\bar{Q})$, the primitives of them are bounded and uniformly continuous on $\bar{Q}$. Hence, for each $z \in\left[u_{0}, v_{0}\right], \mathcal{A}\left(\left[u_{0}, v_{0}\right]\right)$ is equi-uniformly continuous. In view of Ascoli-Arzelà theorem, $\mathcal{A}\left[u_{0}, v_{0}\right]$ is relatively compact.

According to Lemma $4, \mathcal{A}$ has the minimal fixed point $z_{*}$ and the maximal fixed point $z^{*}$. It follows from (3) and (4) that $z_{*}$ and $z^{*}$ are the minimal and maximal solutions of the hyperbolic equation (1).

Remark 1(Ascoli-Arzelà Theorem) If $F$ is bounded and uniformly continuous on $[a, b]$, then exists $\left\{f_{n}\right\} \subset F$ is equi-uniformly continuous on $[a, b]$.

\section{Examples}

In this section, we shall give two examples for the application of Theorem 1.

Example 1 Consider the existence of solutions of the following special hyperbolic equation

$$
\begin{cases}\frac{\partial^{2} z}{\partial x \partial y}=f(x, y, z), & \text { on } Q \\ z(x, 0)=\sigma(x), & 0 \leq x \leq 1, \\ z(0, y)=\tau(y), & 0 \leq y \leq 1,\end{cases}
$$

where $Q=\{(x, y): 0<x<1,0<y<1\}, \bar{Q}$ is the closure of $Q, z \in C(\bar{Q}), C(\bar{Q})$ denotes the space of all continuous functions on $\bar{Q} \cdot \frac{\partial^{2} z}{\partial x \partial y}$ denotes the mixed distributional derivative of $z$ and $f$ is distributionally Henstock-Kurzweil integrable satisfying

$\left(1^{\circ}\right)$ increasing in $z$ for fixed $(x, y) \in Q$,

$\left(2^{\circ}\right)$ bounded, there exist $f_{1}, f_{2} \in D_{H K}(\bar{Q})$ such that $f_{1}(\cdot, \cdot) \leq f(\cdot, \cdot, z) \leq f_{2}(\cdot, \cdot)$, for all $z \in C(\bar{Q})$.

It is well known that the $D_{H K}$-integral contains the Lebesgue integral. Obviously, the existence of solutions of equation (9) is guaranteed by Theorem 1 .

Example 2 Consider another hyperbolic equation

$$
\begin{cases}\frac{\partial^{2} z}{\partial x \partial y}=f\left(x, y, z, \frac{\partial z}{\partial x}, \frac{\partial z}{\partial y}\right)=r(x)+g\left(x, y, z, \frac{\partial z}{\partial x}, \frac{\partial z}{\partial y}\right), & \text { on } Q \\ z(x, 0)=\sigma(x), & 0 \leq x \leq 1, \\ z(0, y)=\tau(y), & 0 \leq y \leq 1,\end{cases}
$$

where $Q=\{(x, y): 0<x<1,0<y<1\}, \bar{Q}$ is the closure of $Q, z \in C(\bar{Q}), C(\bar{Q})$ denotes the space of all continuous functions on $\bar{Q}, r(x)$ is the distributional derivative of $R(x)=\sum_{n=1}^{\infty} \frac{\sin n^{2} \pi t}{n^{2}}, \frac{\partial^{2} z}{\partial x \partial y}$ denotes the mixed distributional derivative of $z$ and $g$ is distributionally Henstock-Kurzweil integrable satisfying

(i) $g\left(x, y, z, \frac{\partial z}{\partial x}, \frac{\partial z}{\partial y}\right)$ is increasing in $z$ for all $(x, y) \in Q$,

(ii) There exist $f_{1}, f_{2} \in D_{H K}(\bar{Q})$ such that $f_{1}(\cdot, \cdot) \leq f\left(\cdot, \cdot, z, \frac{\partial z}{\partial x}, \frac{\partial z}{\partial y}\right) \leq f_{2}(\cdot, \cdot)$, for all $z \in C(\bar{Q})$ and $\frac{\partial z}{\partial x}, \frac{\partial z}{\partial y} \in D_{H K}(\bar{Q})$. 
It is easy to see that $R(x) \in C[0,1]$ and $R(0)=0$, hence $r(x)$ is DHK-integrable. In view of $(10), f\left(x, y, z, \frac{\partial z}{\partial x}, \frac{\partial z}{\partial y}\right)$ satisfies $\left(C_{1}\right),\left(C_{2}\right)$. Thus, for $(x, y) \in Q, z \in C(\bar{Q})$, we have

$$
r(x)+f_{1}(\cdot, \cdot) \leq f\left(\cdot, \cdot, z, \frac{\partial z}{\partial x}, \frac{\partial z}{\partial y}\right) \leq r(x)+f_{2}(\cdot, \cdot)
$$

That is, $f\left(x, y, z, \frac{\partial z}{\partial x}, \frac{\partial z}{\partial y}\right)$ satisfies $\left(C_{3}\right)$.Therefore, the existence of solutions of equation (10) is guaranteed by Theorem 1 .

Remark 2 The Riemann function $R(x)$ in $(10)$ is continuous everywhere in the interval $(0,1)$ within the irrational points but continuous nowhere in the interval $(0,1)$ within the rational points. Then, the distributional derivative $r$ in (10) is neither $H K$ nor Lebesgue integrable. Hence, the methods using HK or Lebesgue integral are ineffective for equation (10).

\section{References}

Alexiewicz, A., \& Orlicz, W. (1956). Some remarks on the existence and uniqueness of solutions of the hyperbolic equation. Studia Math, 156-160. http://dx.doi.org/10.1093/acprof:oso/9780195309836.001.0001

Ang, D. D., Schmitt, K., \& Vy, L. K. (1997). A multidimensional analogue of the Denjoy-Perron-HenstockKurzweil integral. Bull. Belg. Math. Soc., 355-371.

Bugajewski, D., \& Szufla, S. (1995). On the Aronszain property for differential equations and the Denjoy integral. Comment. Math., 61-69.

Cichoń, M., \& Kubiaczyk, I. (2001). Knerser-type theorem for the Darboux problem in Banach spaces. Comment. Math. Univ. Carolinae, 267-279.

Deblasi, F. S., \& Myjak, J. (1986). On the structure of the set of solutions of the Darboux problem for hyperbolic equations. Proc. Edinbourgh Math. Soc., 7-14.

Górniewicz, L., \& Pruszko, T. (1981). On the set of solutions of the Darboux problem for some hyperbolic equations. Bull. Acad. Polon. Sci. Math, 279-285.

Guo, D., Cho, Y. J., \& Zhu, J. (2004). Partial Ordering Methods in Nonlinear Problems. Nova Science Publishers, Inc., Hauppauge, NY. (pp.201-206).

Lee, P. Y. (1989). Lanzhou lectures on Henstock integration. World Scientific, Singapore.

Lu, Y., Ye, G., Wang, Y., \& Liu, W. (2011). Existence of Solutions of the Wave Equation Involving the Diatributional Henstock-Kurzweil Integral. Differental Integral Equations, 1063-1071.

Rzepecki, B. (1986). On the existence of solutions of the Darboux problem for the hyperbolic partial differential equations in Banach spaces. Rend. Sem. Mat. Uni. Padova, 201-206.

Schwabik, Š., \& Ye, G. (2005).Topics in Banach space integration. World Scientific, Singapore.

\section{Copyrights}

Copyright for this article is retained by the author(s), with first publication rights granted to the journal.

This is an open-access article distributed under the terms and conditions of the Creative Commons Attribution license (http://creativecommons.org/licenses/by/3.0/). 